benefit is to be gained both for the patient and the doctor, particularly in the 'wee small hours'.
A. W. HORROCKS
Consultant Radiologist,
Wythenshawe Hospital,
Manchester, England

\title{
REFERENCE
}

Keats T. (1988) Atlas of normal roentgen variants that may simulate disease 4th edn. Wolfe year book.

\section{Clinical and Resuscitative Data}

By R. P. H. Dunhill \& M. P. Colvin

Blackwell Scientific Publications, Oxford, 1989. 248 pp. $£ 16.95$.

This is the fourth edition of a book with which I must admit to be unfamiliar. It has been intended to provide essential data and information to assist doctors in resuscitation. It is apparently aimed particularly at the accident and emergency department.

There are many old chestnuts in the book which detract from its usefulness. For example, the calculation of correction of metabolic acidosis by the base deficit $\times \frac{1}{3}$ of the weight in $\mathrm{kg}$ to give you the mmol of bicarbonate is not helpful in many resuscitation episodes and for example after cardiac arrest this would now be considered totally inappropriate. Such isolated information without background discussion may mislead the inexperienced. However, there are many nuggets of information particularly related to anaesthesia which I found extremely useful. I was pleasantly surprised to find such a good section on local and regional anaesthesia with some excellent illustrations.

When the book strayed into critical care resuscitation it met some fundamental problems. For assessing and resuscitating patients with multiple injuries there are welldocumented tried-and-tested protocols. Any modern text must now refer to ATLS. The personal approach of the authors could not be recommended in many modern accident and emergency departments, including my own. There is little practical help to be found and some potentially misleading unqualified advice. The administration of inotropic agents without the measurement of cardiac output and systemic vascular resistance is not the best practice-to advise this in the seriously injured cannot be justified.

If the section on major trauma is unhelpful then the section on cardiac arrest is worse. The Resuscitation Council have produced clear guidelines based on current European and North American consensus and if followed prevent idiosyncratic and unjustifiable recommendations, as $150 \mathrm{mEq}$ of bicarbonate at the start of cardiac arrest.

It is such a pity that the section on critical care is so out-of-date when there is so much useful information in this book. The chart on dosage of drugs delivered by infusion is almost worth buying the book for. The section on critical care must be put in context 
against the chapter on the Management of Acute Renal Failure which appears to the non-specialist as being of much more value.

The section on drug doses is excellent, although in fairness much of the information is available in the National Formulary.

In summary there is a lot of useful information in this book but it is seriously flawed by the section on cardiac arrest and multiple injuries. I appreciate that it is only a book intended to give resuscitative data and not a book on resuscitation itself and for these very reasons these sections should not have been included. I have doubts whether $£ 17.00$ is a reasonable investment for the other information contained in this book.

\section{A. D. REDMOND}

South Manchester Accident and Emergency Service, Withington Hospital, Manchester, England

\section{JOURNAL REVIEW}

\section{Surgical Infection}

Surgical Infection Study Group, 1989.

This is a new journal which will be circulated free of charge every 6 months. It is prepared by members of the recently formed Surgical Infection Study Group. The aim is to review the papers published in the preceding 6 months concerning all aspects of infection in general surgery, orthopaedics, urology, gynaecology and obstetrics. Eacto section contains commentaries (short reviews of papers considered to be of particular importance) and annotated references (often one-two sentences long) of many other papers. When I first opened it I did so with some scepticism. However, I found it excellent reading and I am sure that I shall find the annotated references particularly useful in the future.

D. E. F. TWEEDLE

EDITOR,

Surgical Infection, Vale of Leven General District Hospital, Alexandria, Dunbartonshire, Scotland 